Come rompere la routine e lavorare le competenze orali in classe di FLE raccontando una bella storia

\title{
Luigi Zammartino
}

\section{(2) OpenEdition \\ Journals}

Edizione digitale

URL: http://journals.openedition.org/esp/1509

DOI: 10.4000/esp.1509

ISSN: 2532-0319

Editore

Centre d'Information sur l'Éducation Bilingue et Plurilingue

\section{Edizione cartacea}

Data di pubblicazione: 1 dicembre 2017

Paginazione: 45-52

ISSN: 1127-266X

Notizia bibliografica digitale

Luigi Zammartino, "Come rompere la routine e lavorare le competenze orali in classe di FLE

raccontando una bella storia », Éducation et sociétés plurilingues [Online], 43 | 2017, Messo online il 01

février 2019, consultato il 15 septembre 2020. URL : http://journals.openedition.org/esp/1509 


\section{COME ROMPERE LA ROUTINE E LAVORARE LE COMPETENZE ORALI IN GLASSE DI FLE RACGONTANDO UNA BELLA STORIA}

\section{Luigi ZAMMARTINO}

L'attention et la mémoire sont deux clefs de tout apprentissage. Travailler l'expression orale en approche communicative - à travers la narration d'histoires actives fondées sur les critères de questions posées, de prises de notes, de chevauchements de parole et interruptions - permet de développer l'attention, d'enrichir les productions langagières et d'évaluer les élèves sous une forme ludique. L'article propose une séance didactique d'une heure dans le cadre de l'enseignement du français deuxième langue vivante à des collégiens italiens de niveau débutant. Dans cette méthode, pratiques et contenus interagissent, ils sont sans cesse réflexifs l'un par rapport à l'autre, tout en favorisant la participation équilable des élèves el la progression des compélences d'interaction verbale, qui représente l'un des aspects les plus importants de l'acquisition d'une langue étrangère.

Mots clés: Narration d'histoires, français langue étrangère en Italie, questionnaire, activité d'enseignement et d'apprentissage, collégiens.

Concentration and memory are two keys to language learning. Working on oral expression in a communicative approach - telling lively stories by asking questions, taking notes, listening to everyone talk at the same time and interrupting - allowes developing pupils' concentration, enriching their verbal productions and evaluating them in a playful way. The article proposes one hour of didactics in a class of French as a foreign, living language, with Italian beginners in a secondary school. In this method, practices and content go together, constantly mirroring each other while insuring each pupil's participation and improving their capacity to interact, which is one of the most valuable competences of foreign language acquisition.

Keyzerds: Telling stories, French as a foreign language in italiana, questionnaire, teaching and learning, secondary school pupils.

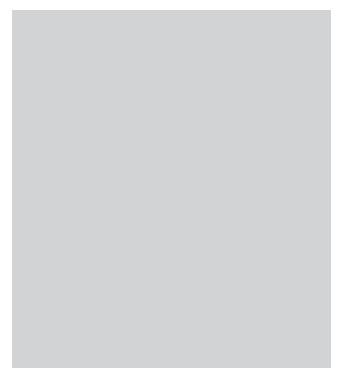

0 torie e racconti partecipano attivamente alla costruzione della personalità dei nostri apprendenti e sono anche eccellenti mezzi pedagogici per il raggiungimento, sotto forma ludica, di obiettivi prestabiliti. Da anni lavoro le competenze orali della L2 lingua francese nelle classi della Secondaria di Primo Grado raccontando storielle, ma l'idea di questo articolo nasce solo in seguito ad un atelier sul tema che ho animato in qualità di formatore dei formatori. Da un'indagine - circa 150 questionari somministrati ex post a formatori FLE di ogni ordine (1) - risulta in percentuale maggiore che in classe si lavora l'orale a partire 
Come rompere la routine e lavorare le competenze orali in classe di FLE raccontando una bella storia

L. ZAMMARTINO dalla comprensione di un testo scritto o attraverso uno scambio di domande e risposte tra insegnante e alunno o tra alunno e alunno, con la conseguenza che i discenti hanno spesso difficoltà a trovare le giuste parole e sono alquanto impacciati a comunicare in pubblico. Le prolungate pause dovute alle esitazioni dell'alunno che tarda ad esprimere la sua preferenza tra possibili soluzioni ad una domanda non stimolano l'apprendimento attivo, né un'autentica partecipazione alla lezione, contribuendo a distrarre e demotivare l'intera classe. Risulta altresì dall'indagine condotta, che oltre l' $80 \%$ degli insegnanti intervistati non racconta storie spontanee in classe - magari anche inventate al momento - per arricchire e contestualizzare il lessico, la sintassi, la grammatica precedentemente affrontati, allo scopo di ripasso e favorire la didattica dell'orale; insomma non si dà voce a brevi e divertenti racconti, per fare della lingua straniera una cosa viva, aperta all'incontro e strumento di comunicazione prima che di studio, ma ci si affida comunemente al manuale e ai suoi esercizi interattivi. Eppure, senza effetti speciali, ma con le semplici modalità di seguito riportate, $\mathrm{i}$ docenti che hanno partecipato alla giornata di formazione, hanno giudicato la mia attività didattica di «ascoltare storielle e rispondere alle sollecitazioni del narratore» molto coinvolgente:

- diviser la classe en trois équipes

- expliquer le brouillon de prise de notes

- expliquer comment répondre aux questions

- présenter le règlement du tour de parole

- valider les points

La sana competizione è un mezzo utile per stimolare la motivazione ad apprendere. Far parte di una équipe valorizza e responsabilizza il discente. Dividendo pertanto la classe in 3 squadre, Bleu, Blanc e Rouge ad esempio, si permette al singolo alunno di portare avanti un progetto comune: «comprendere il più possibile la narrazione, per rispondere in maniera esatta al maggior numero di domande che di volta in volta verranno poste, al fine di contribuire al successo della squadra». Prendere appunti è anch'esso un passaggio fondamentale ed è compito dell'insegnante chiarirlo prima dell'inizio di ogni storia. Fissare nomi, numeri e passaggi cruciali del racconto, aiuta molto a ricordare, e farlo senza preoccuparsi delle regole scritte, induce l'apprendente a concentrare le sue attenzioni tutte sull'ascolto. Si dirà inoltre, prima di dare inizio al racconto, che lo stesso segue un andamento guidato e, poiché sotto forma di ridondanze e di ripetizioni saranno sottolineati i suoi momenti topici, l'apprendente-uditore avrà più di un'occa- 
Come rompere la routine e lavorare le competenze orali in classe di FLE raccontando una bella storia

L. ZAMMARTINO sione per appuntarli. Questo non significa affatto che il racconto viene narrato più volte, ma che la narrazione avviene con frasi che si collegano tra di loro con una progressione che aggiunge di volta in volta dei dati, con differenze nel tono e nell'inflessione della voce, e con la capacità di specificare meglio le sensazioni con espressioni più pertinenti, centrando l'obiettivo di cogliere maggiori dettagli. Cruccio di noi insegnanti di Langue Vivante è anche l'ostinazione dei nostri alunni nel rispondere con monosillabi. Appunto per questo, è importante spiegare in modo chiaro come rispondere alle varie domande, rispettando sempre l'ordine degli elementi: soggetto, verbo e complemento.

Tipologie di domande e risposte a narrazione conclusa:

- Formulata con Est-ce que, rispondere Oui o Non, e completare la risposta.

- Formulata con C'est vrai o C'est faux, rispondere semplicemente Oui, c'est vrai, oppure Non, c'est faux.

- Formulata su di un'ellissi narrativa, rispondere On ne sait pas.

Allenare gli apprendenti della L2 lingua francese a rispondere VRAI o FAUX, come a riconoscere le omissioni narrative rispondendo ON NE SAIT PAS, si avvezzano gli stessi alle procedure degli esami DELF (2) per il raggiungimento delle competenze linguistiche definite secondo i livelli proposti dal Quadro Europeo Comune di Riferimento per le Lingue (QEGRL). Anche il «turno di parola» è importante e farà parte di quel numero di regole da considerare. Prima di iniziare la narrazione è fondamentale che i nostri ragazzi abbiano chiaro quali regole devono rispettare e quali sanzioni sono previste per i trasgressori, così come quali sono i premi o i vantaggi per coloro che si comportano bene. Pertanto, la convalida dei punti è parte integrante dell'attività proposta. Si decide 1 punto per ogni risposta completa ed esatta, $1 / 2$ punto per chi risponde esattamente senza rispettare la formulazione completa, e il punto passa all'altra équipe se il turno di parola è disatteso. Sulle regole pattuite non saranno ammesse eccezioni.

L'obiettivo primario dell'attività proposta è la partecipazione attiva della classe. Per permettere ciò ho affinato negli anni la tecnica e sono giunto alla conclusione che «il numero delle domande dovrà essere pari al numero dei componenti delle squadre». Solo in tal modo si è certi che tutti siano coinvolti. Essendo un'attività di squadra, è compito dei componenti la squadra stessa decidere chi dovrà rispondere alle domande più difficili o a quelle più tarate. $\grave{\mathrm{E}}$ ammesso altresì un tutor per chi è confuso o demotivato, e questa risorsa aiuterà tutti a crescere. Un'attività didattica funzio- 
Come rompere la routine e lavorare le competenze orali in classe di FLE raccontando una bella storia

L. ZAMMARTINO na del resto se in classe tutti sono coinvolti e non solo quelli più bravi o brillanti!

Ecco un esempio di attività narrativa svolta nelle classi prime (età media dei discenti 10/11 anni), periodo fine gennaio, della Secondaria di Primo Grado. Il francese nel curricolo d'Istituto è seconda lingua straniera, per un monte ore annuale di 66, suddivise in 2 ore settimanali; il livello linguistico è quello principiante. L'attività proposta tiene conto delle conoscenze pregresse dei discenti quali: se présenter et présenter quelqu'un; les parties du corps; la famille; les couleurs... Partendo da tali prerequisiti, lo scopo principale dell'attività è guidare lo studente verso un apprendimento autonomo:

Dans cette histoire il y a deux personnages: un garçon et une fille! Le garçon s'appelle Antoine, ... la fille Assuntina. Vous avez déjà très bien compris que le garçon, il est français et que la fille, elle est napolitaine! Antoine a vingtdeux ans, il est français, oui c'est vrai il est français ... mais attention il habite Naples et il est chef dans le restaurant de sa famille. Sa famille se compose de cinq personnes, c'est-à-dire sa mère, son père, deux seurs et lui. Antoine aime beaucoup sa famille et son travail! Assuntina a dix-sept ans et 364 jours, elle, demain, aura dix-huit ans! L'anniversaire d'Assuntina arrive demain, c'est-à-dire le 8 juillet!! À l'occasion, son père, lui organise une grande fête, une immense fête, 290 invités plus ou moins! Oh là là... 290 invités... Assuntina est petite et mince, elle est très sportive: elle fait du tennis, de la natation et de l'équitation. Antoine ne pratique pas de sport! Antoine est grand et robuste, il a les yeux verts et les cheveux courts et noirs! Les cheveux d'Assuntina sont blonds et longs. Ses yeux sont bleus, bleus clairs comme la mer! Et ... c'est à la mer qu'on fête l'anniversaire d'Assuntina! Une belle et grande fête de 290 invités... c'est au restaurant d'Antoine que le père d'Assuntina a tout organisé pour la fête de dix-huit ans de sa fille! Voilà... l'histoire prend du terrain... Au restaurant les invités mangent, ils mangent beaucoup; dansent, ils dansent longtemps: chantent-ils?' Ils chantent beaucoup! Il fait beau, il fait chaud ... et dans le noir le gâteau arrive. Un gâteau à cing étages au chocolat. Un, deux, trois, quatre, cinq étages... oh là là... Les 18 chandelles roses sont allumées ... et le beau visage d'Antoine qui porte le gâteau s'illumine ... ses yeux verts s'illuminent, ses cheveux noirs s'illuminent ... Antoine porte le gâteau et tout le monde chante: bon anniversaire Assuntina ... bon anniversaire à toi ... mais ... attention Assuntina est hypnotisée ... elle regarde Antoine, elle regarde ses yeux verts... et Antoine, lui aussi, il est hypnotisé: il regarde Assuntina, il regarde ses yeux bleus clairs, il regarde ses cheveux blonds. BOOM ... pour regarder fixement Assuntina, Antoine trébuche ... et le gâteau tombe sur la tête du père d'Assuntina ... Oh mon Dieu ... le chocolat est partout ... sur sa tête, dans ses oreilles, sur son nez ... partout!! Pour l'instant la narration s'arrête... 
Come rompere la routine e lavorare le competenze orali in classe di FLE raccontando una bella storia

L. ZAMMARTINO
Per avere un clima disteso e partecipativo, per migliorare l'attenzione e la comprensione, bisogna sfruttare al massimo tutti i margini di autonomia che l'insegnante ha a sua disposizione; pertanto la narrazione di storielle, per lavorare le competenze orali, in classe deve essere supportata da:

- Du non verbal

- De la voix

- Des pauses

- Des silences

- Des regards

Gli studenti prestano molta attenzione ai nostri gesti e ai nostri movimenti. Giocare con la voce è uno dei nostri strumenti principali: spezza il ritmo della lezione e rompe la monotonia. Le nostre storie devono essere supportate quindi da stratagemmi di comunicazione non verbale, da sorrisi, da occhiate rasserenanti. Devono essere sostenute da gesti metaforici che descrivano i contenuti semantici che si stanno narrando o semplicemente corredate di gesti ritmici di battuta che scandiscano aspetti particolari del discorso. Le pause e i silenzi sono molto significativi ed è importante farli apprendere! Ma soprattutto le nostre storie devono avere l'aria d'essere improvvisate! Non bisogna leggerle: evitare di tuffare il capo nel testo - per intenderci - vuol dire molto, molto più di quanto non si pensi! Inoltre, raccontiamo solo storie divertenti; talvolta basta davvero poco per catturare l'attenzione. Se la protagonista della nostra storia fosse stata Marie-France o Chantal, cliché dei nostri manuali, avrebbe suscitato in una classe della provincia napoletana lo stesso interesse di una popolarissima Assuntina? Nelle nostre storie dunque facciamo entrare il retaggio dei nostri alunni: ritrovarsi nei racconti è il modo migliore per avvicinarsi e partecipare!

Alla fine della narrazione facciamo rimbalzare alla lavagna interattiva le varie domande:

Comment s'appelle le père d'Antoine?

C'est vrai qu'il fait mauvais temps?

Combien de personnages comporte l'histoire?

Comment se compose la famille d'Antoine?

Antoine a-t-il des frères?

De quelle couleur sont les cheveux d'Assuntina?

Quel âge a Antoine?

Quand arrive l'anniversaire d'Antoine?

Quand arrive l'anniversaire d'Assuntina?

Quel est le nombre approximatif des invités?

Le restaurant se trouve-t-il à la montagne? 
Come rompere la routine e lavorare le competenze orali in classe di FLE raccontando una bella storia

L. ZAMMARTINO
Où habite Antoine?

Antoine, est-ce qu'il est content d'être chef?

C'est vrai que les yeux d'Assuntina sont marron?

Comment s'appelle le restaurant?

Etc. etc. etc.

Questa attività didattica permette all'insegnante una verifica orale completa (ascolto, comprensione e produzione) di ripasso dei principali argomenti trattati, coinvolgendo l'intera classe e impiegando un'unica ora di lezione, ma è anche un punto di partenza per la messa in opera di una quantità di successive esercitazioni interessanti all'interno di una classe o di consegne per il lavoro a casa. Se ad esempio in classe si è lavorato esclusivamente sulle competenze orali, a casa, viceversa, gli alunni potranno esercitarsi su quelle scritte, operando una breve produzione che va dalla semplice ma simpatica ricerca di «Donnez un titre au conte» oppure "Rédigez un texte bref»" magari indicando il numero delle parole minime o massime da impiegare per fantasticare sul finale della storia! 
Come rompere la routine e lavorare le competenze orali in classe di FLE raccontando una bella storia

L. ZAMMARTINO

Se desideriamo far sviluppare le competenze linguistiche ai nostri alunni, bisogna che il discente sia attivo nei suoi apprendimenti, vale a dire che le attività che noi insegnanti gli proponiamo abbiano del senso per lui e lo motivino a comunicare in L2. Raccontare storie e racconti, e dare soprattutto l'impressione che questi nascano e crescano dal nulla, porta gli alunni a familiarizzare con la pronuncia e la prosodia. Le strutture grammaticali presentate in questo contesto ludico, e ripetute ad oltranza, permettono il loro apprendimento in maniera implicita, la loro appropriazione e la loro memorizzazione senza noia. Raccontare storielle, per di più, fa sì che la comprensione linguistica avvenga soprattutto attraverso un canale emotivo e che il godimento promosso dall'ascolto lasci abbassare il filtro affettivo, con la conseguenza che quel sentimento di frustrazione generato di norma dal fatto di non conoscere il significato di tutte le singole parole sia in parte annullato. Infine, raccontare permette all'alunno di reperire degli indizi visuali e uditivi. Nella lingua materna un gran numero di questi sono necessari per giungere a diversi stadi di comprensione. Durante una sequenza di lingua straniera, per arrivare al senso l'alunno avrà bisogno dapprima di questi aiuti extra-linguistici, e tali possono essere dei gesti, delle particolari intonazioni effettuate dall'insegnane, o altrettanti espedienti che diano vita alla storia e che la rendono comprensibile.

\section{NOTE}

(1) I questionari sono stati somministrati nell'ambito della giornata di formazione $A U$ FIL DU FLE, 16/11/2016, organizzata dalla Associazione culturale italo-francese Francofil, in partenariato con l'Institut français-Italia e l'Institut français-Napoli, formazione rivolta a docenti di lingua francese degli istituti di ogni ordine e grado e docenti di discipline non linguistiche (DNL) secondo la metodologia CLIL/EMILE della regione Campania.

(2) Gli esami DELF (Diplôme d'études en langue française) e DALF (Diplôme approfondi de langue française) sono composti da 6 diplomi indipendenti, di difficoltà progressiva, che corrispondono ai 6 livelli del Quadro Europeo Comune di Riferimento per le Lingue (QECR) prodotto dal Consiglio d'Europa. Sono 4 diplomi per il DELF e 2 per il DALF, ottenibili separatamente l'uno dall'altro, anche in centri d'esami diversi. Il DELF e il DALF sono costituiti da 4 prove che valutano le 4 competenze: comprensione e espressione orale, comprensione e espressione scritta. Entrambe le certificazioni sono sottoposte all'autorità di una specifica Commissione nazionale con sede presso il CIEP (Centre International d'Études Pédagogiques). I diplomi DELF e DALF sono senza limiti di validità e riconosciuti a livello internazionale. 